\title{
Re-ionization of the IGM - massive stars versus QSOs
}

\author{
Piero Madau \\ Astronomy and Astrophysics Department, University of California, \\ 477 Clark Kerr Hall, Santa Cruz, CA 95064, USA
}

\begin{abstract}
In popular cold dark matter cosmological scenarios, stars may have first appeared in significant numbers around a redshift of 10 or so, as the gas within protogalactic halos with virial temperatures $T_{\text {vir }} \gtrsim 20000 \mathrm{~K}$ (corresponding to masses comparable to those of present-day dwarf ellipticals) cooled rapidly due to atomic processes and fragmented. It is this 'second generation' of subgalactic stellar systems, aided perhaps by an early population of accreting black holes in their nuclei, which may have generated the ultraviolet radiation and mechanical energy that ended the cosmic 'dark ages' and reheated and re-ionized most of the hydrogen in the universe by a redshift of $z=6$. The detailed history of the universe during, and soon after these crucial formative stages, depends on the power-spectrum of density fluctuations on small scales and on a complex network of poorly understood feedback mechanisms, and is one of the missing links in galaxy formation and evolution studies.
\end{abstract}

\section{Introduction}

At epochs corresponding to $z \approx 1000$ the intergalactic medium (IGM) is expected to recombine and remain neutral until sources of radiation and heat develop that are capable of re-ionizing it. The detection of transmitted flux shortward of the Ly $\alpha$ wavelength in the spectra of sources at $z \gtrsim 5$ implies that the hydrogen component of this IGM was ionized at even higher redshifts. The increasing thickening of the Ly $\alpha$ forest recently measured in the spectra of SDSS $z \simeq 6$ quasars (Becker et al. 2001; Djorgovski et al. 2001) may be the signature of the trailing edge of the cosmic re-ionization epoch. It is clear that substantial sources of ultraviolet photons and mechanical energy, like young star-forming galaxies, were already present back then. The re-ionization of intergalactic hydrogen at $z \gtrsim 6$ is unlikely to have been accomplished by quasi-stellar sources: the observed dearth of luminous optical and radio-selected QSOs at $z>3$ (Shaver et al. 1996; Fan et al. 2001), together with the detection of substantial Lyman-continuum flux in a composite spectrum of Lyman-break galaxies at $\langle z\rangle=3.4$ (Steidel et al. 2001), both may support the idea that massive stars in galactic and subgalactic systems - rather than quasars - re-ionized the hydrogen component of the IGM when the universe was less than $5 \%$ of its current age, and dominate the 1 Ryd metagalactic flux at all redshifts $z>3$. An episode of pregalactic star formation may also provide a possible explanation for the widespread existence of heavy elements (like carbon, oxygen, and silicon) in the IGM. There is mounting evidence that the double re-ionization of helium may have occurred later, at a 
redshift of $z=3$ or so (e.g., Kriss et al. 2001): this is likely due to the integrated radiation emitted above 4 Ryd by QSOs.

Establishing what ended the dark ages and when is important for determining the impact of cosmological re-ionization and reheating on several key cosmological issues, from the role re-ionization plays in allowing protogalactic objects to cool and make stars, to determining the thermal state of baryons at high redshifts and the small-scale structure in the temperature fluctuations of the cosmic microwave background. Conversely, probing the re-ionization epoch may provide a means for constraining competing models for the formation of cosmic structures. For example, popular modifications of the cold dark matter (CDM) paradigm that attempt to improve over CDM by suppressing the primordial power-spectrum on small scales, like warm dark matter (WDM), are known to reduce the number of collapsed halos at high redshifts and make it more difficult to re-ionize the universe (Barkana et al. 2001). Similarly, 'feedback' processes like early photo-ionization may prevent gas cooling and inhibit star formation in the majority of the dwarf subhalos that are predicted to populate the virialized extent of a Milky Way's halo in CDM cosmogonies (Moore et al. 1999); the dark matter satellites of the Milky Way would still be present, but only a small fraction of them would actually be visible, having formed stars prior to the reheating of the IGM (Bullock et al. 2000). In this talk I will summarize some recent theoretical developments in understanding the astrophysics of the epoch of first light and the impact that some of the earliest generations of stars, galaxies, and black holes in the universe may have had on the IGM.

\section{Re-ionization by massive stars}

In a CDM universe, structure formation is a hierarchical process in which non linear, massive structures grow via the merger of smaller initial units. Large numbers of low-mass galaxy halos are expected to form at early times in these popular theories, leading to an era of re-ionization, reheating, and chemical enrichment. Most models predict that intergalactic hydrogen was re-ionized by an early generation of stars or accreting black holes at $z=7-15$. One should note, however, that while numerical $N$-body + hydrodynamical simulations have convincingly shown that the IGM does fragment into structures at early times (e.g., Cen et al. 1994; Zhang et al. 1995; Hernquist et al. 1996), the same simulations are much less able to predict the efficiency with which the first gravitationally collapsed objects lit up the universe at the end of the dark age.

The scenario that has received the most theoretical studies is one where hydrogen is photo-ionized by the UV radiation emitted either by quasars or by stars with masses $\gtrsim 10 \mathrm{M}_{\odot}$ (e.g., Shapiro \& Giroux 1987; Haiman \& Loeb 1998; Madau et al. 1999; Chiu \& Ostriker 2000; Ciardi et al. 2000), rather than ionized by collisions with electrons heated up by, e.g., supernova-driven winds from early pregalactic objects. In the former case a high degree of ionization requires about $13.6 \times\left(1+t / \bar{t}_{\text {rec }}\right) \mathrm{eV}$ per hydrogen atom, where $\bar{t}_{\text {rec }}$ is the volumeaveraged hydrogen recombination timescale, $t / \bar{t}_{\text {rec }}$ being much greater than unity already at $z \simeq 10$ according to numerical simulations. Collisional ionization to a neutral fraction of only a few parts in $10^{5}$ requires a comparable energy input, i.e., an IGM temperature close to $10^{5} \mathrm{~K}$ or about $25 \mathrm{eV}$ per atom. 
Massive stars will deposit both radiative and mechanical energy into the interstellar medium of protogalaxies. A complex network of feedback mechanisms is likely at work in these systems, as the gas in shallow potential is more easily blown away (Dekel \& Silk 1986; Tegmark et al. 1993; Mac Low \& Ferrara 1999; Mori et al. 2002), thereby quenching star formation. Furthermore, as the blastwaves produced by supernova explosions reheat the surrounding intergalactic gas and enrich it with newly formed heavy elements (Madau et al. 2001), they can inhibit the formation of nearby low-mass galaxies due to 'baryonic stripping' (Scannapieco et al. 2002). It is therefore difficult to establish, whether an early input of mechanical energy will actually play a major role in determining the thermal and ionization state of the IGM on large scales. While during the evolution of a 'typical' stellar population more energy is lost in ultraviolet radiation than in mechanical form, the relative importance of photo-ionization vs. shock ionization will depend on the efficiency with which radiation and mechanical energy actually escape into the IGM. Consider, for example, the case of an early generation of subgalactic systems collapsing at redshift $z=9$ from 2- $\sigma$ fluctuations. At these epochs their dark matter halos would have virial radii $r_{\mathrm{vir}}=0.75 \mathrm{~h}^{-1} \mathrm{kpc}$ and circular velocities $v_{\mathrm{c}}\left(r_{\mathrm{vir}}\right)=25 \mathrm{~km} \mathrm{~s}^{-1}$, corresponding in top-hat spherical collapse to a virial temperature $T_{\mathrm{vir}}=0.5 \mu \mathrm{m}_{\mathrm{p}} v_{\mathrm{c}}^{2} / k \simeq 10^{4.3} \mathrm{~K}$ and halo mass $M=0.1 v_{\mathrm{c}}^{3} / \mathrm{G} H \simeq 10^{8} h^{-1} \mathrm{M}_{\odot}{ }^{1}$.

Halos in this mass range are characterized by very short dynamical timescales (and even shorter gas cooling times due to atomic hydrogen) and may therefore form stars in a rapid but intense burst before SN feedback quenches further star formation. For a star formation efficiency of $f=0.1, \Omega_{b} h^{2}=0.02^{2}, h=0.5$, and a Salpeter IMF, the explosive output of 10000 SNe will inject an energy $E_{0} \approx 10^{55} \mathrm{erg}$. This is roughly a hundred times higher than the gas binding energy: a significant fraction of the halo gas will then be lifted out of the potential well ('blow-away') and shock the intergalactic medium (Madau et al. 2001). If the explosion occurs at cosmic time $t=4 \times 10^{8} \mathrm{yr}$ (corresponding in the adopted cosmology to $z=9$ ), at time $\Delta t=0.4 t$ after the event, it is a good approximation to treat the cosmological blast wave as adiabatic, with proper radius given by the standard Sedov-Taylor self-similar solution,

$$
R_{s} \simeq\left(\frac{12 \pi \mathrm{G} \eta E_{0}}{\Omega_{b}}\right)^{1 / 5} t^{2 / 5} \Delta t^{2 / 5} \simeq 17 \mathrm{kpc}
$$

Here $\eta \approx 0.3$ is the fraction of the available SN energy that is converted into kinetic energy of the blown-away material (Mori et al. 2002). At this instant the shock velocity relative to the Hubble flow is $v_{\mathrm{s}} \approx 2 R_{\mathrm{s}} / 5 \Delta t \approx 40 \mathrm{~km} \mathrm{~s}^{-1}$, lower than the escape velocity from the halo center. Behind the shock a shell forms containing a large fraction of the halo gas that has been swept-up during the evolution; its interior is filled with warm $\left(T \lesssim 10^{6} \mathrm{~K}\right)$ gas at a very low density $n \lesssim 10^{-4} \mathrm{~cm}^{-3}$, i.e., slightly below the mean value for the IGM (Mori et al. 2002). The gas temperature just behind the shock front is $T_{\mathrm{s}}=3 \mu m_{\mathrm{p}} v_{\mathrm{s}}^{2} / 16 k \gtrsim 10^{4.3} \mathrm{~K}$,

\footnotetext{
${ }^{1}$ This assumes an Einstein-de Sitter universe with Hubble constant $\mathrm{H}_{0}=100 h \mathrm{~km} \mathrm{~s}^{-1} \mathrm{Mpc}^{-1}$.

${ }^{2}$ Here $\Omega_{b}$ is the baryon density parameter, and $f \Omega_{b}$ the fraction of halo mass converted into stars.
} 


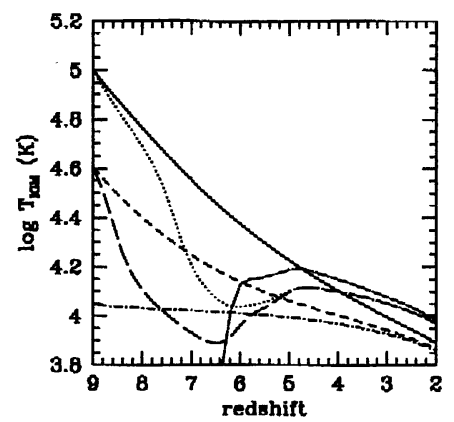

Figure 1. Thermal history of intergalactic gas at the mean density in an Einstein-de Sitter universe with $\Omega_{b} h^{2}=0.02$ and $h=0.5$. Short dash-dotted line: temperature evolution when the only heating source is a constant ultraviolet (CUV) background of intensity $10^{-22}$ ergs cm-2 $\mathrm{s}^{-1} \mathrm{~Hz}^{-1} \mathrm{sr}^{-1}$ at 1 Ryd and power-law spectrum with energy slope $\alpha=1$. Long dash-dotted line: same for the time-dependent quasar ionizing background as computed by Haardt \& Madau (1996: HM). Short-dashed line: heating due to a CUV background but with an initial temperature of $4 \times 10^{4} \mathrm{~K}$ at $z=9$ as expected from an early era of pregalactic outflows. Long-dashed line: same but for a HM background. Solid line: heating due to a CUV background but with an initial temperature of $10^{5} \mathrm{~K}$ at $z=9$. Dotted line: same but for a HM background.

enough to ionize all incoming intergalactic hydrogen. According to the PressSchechter formalism, the co-moving abundance of collapsed dark halos with mass $M=10^{8} h^{-1} \mathrm{M}_{\odot}$ at $z=9$ is $d n / d \ln M \simeq 80 h^{3} \mathrm{Mpc}^{-3}$, corresponding to a mean proper distance between neighboring halos of $\sim 15 h^{-1} \mathrm{kpc}$. With the assumed star formation efficiency, only a small fraction, about $4 \%$, of the total stellar mass inferred today (Fukugita et al. 1998) would actually form at these early epochs. Still, our simple analysis shows that the blast waves from such a population of pregalactic objects could drive vast portions of the IGM to a significantly higher adiabat, $T_{\mathrm{IGM}} \approx 10^{5} \mathrm{~K}$, than expected from photo-ionization, so as to 'choke off' the collapse of further $M \lesssim 10^{9} h^{-1} \mathrm{M}_{\odot}$ systems by raising the cosmological Jeans mass. In this sense the process may be self-regulating.

The thermal history of expanding intergalactic primordial gas at the mean density is plotted in Figure 1 as a function of redshift for a number of illustrative cases. The code we have used includes the relevant cooling and heating processes and follows the non-equilibrium evolution of hydrogen and helium ionic species in a cosmological context. The gas is allowed to interact with the CMB through Compton cooling and either with a time-dependent QSO ionizing background as computed by Haardt \& Madau (1996) or with a time-independent metagalactic flux of intensity $10^{-22} \mathrm{ergs} \mathrm{cm}^{-2} \mathrm{~s}^{-1} \mathrm{~Hz}^{-1} \mathrm{sr}^{-1}$ at $1 \mathrm{Ryd}$ (and power-law spectrum with energy slope $\alpha=1$ ). The temperature of the medium at $z=9-$ where we start our integration - has been either computed self-consistently from photo-heating or fixed to be in the range $10^{4.6-5} \mathrm{~K}$ expected from SNdriven bubbles with significant filling factors. The various curves show that the temperature of the IGM at $z=3-4$ will retain little memory of an early era of 
pregalactic outflows and preheating, and be consistent with that expected from photo-ionization.

It is clear then that SN-driven pregalactic outflows may be an efficient mechanism for spreading metals around. The collective explosive output of about ten thousands SNe per $M \gtrsim 10^{8} h^{-1} \mathrm{M}_{\odot}$ halo at these early epochs could then pollute the entire intergalactic space to a mean metallicity $\langle Z\rangle=\Omega_{Z} / \Omega_{b} \gtrsim 0.003$ (comparable to the levels observed in the $\operatorname{Ly} \alpha$ forest at $z \approx 3$ ) without much perturbing the IGM hydrodynamically, i.e., producing large variations of the baryons relative to the dark matter. The significance of these ideas goes well beyond early metal enrichment. This is because, since the cooling time of collisionally ionized high density gas in small halos at high redshifts is much shorter than the then Hubble time, virtually all baryons are predicted to sink to the centers of these halos in the absence of any countervailing effect (White \& Rees 1978). Efficient feedback is then necessary in hierarchical clustering scenarios to avoid this 'cooling catastrophe', i.e., to prevent too many baryons from turning into stars as soon as the first levels of the hierarchy collapse. The required reduction of the stellar birthrate in halos with low circular velocities may naturally result from the heating and expulsion of material due to OB stellar winds and repeated $\mathrm{SN}$ explosions from the first burst of star formation.

\section{Probing the dark ages with Ly $\alpha$ emitters}

Prior to complete re-ionization at redshift $z_{\mathrm{r}}$, sources of ultraviolet radiation will be seen behind a large column of intervening gas that is still neutral. In this case, because of scattering off the line-of-sight due to the diffuse neutral IGM, the spectrum of a source at $z_{\mathrm{em}}>z_{\mathrm{r}}$ should show the red damping wing of the Gunn-Peterson (1965, herefater GP) absorption trough at wavelengths longer than the local Ly $\alpha$ resonance, $\lambda_{\text {obs }}>\lambda_{\alpha}\left(1+z_{\mathrm{em}}\right)$, where $\lambda_{\alpha}=\mathrm{c} \nu_{\alpha}=1216 \AA$. At $z_{\mathrm{em}} \gtrsim 6$, this characteristic feature extends for more than $1500 \mathrm{~km} \mathrm{~s}^{-1}$ to the red of the resonance, and may significantly suppress the Ly $\alpha$ emission line in the spectra of the first generation of objects in the universe. Measuring the shape of the absorption profile of the damping wing could provide a determination of the density of the neutral IGM near the source (Miralda-Escudé 1998).

A number of authors (Madau \& Rees 2000; Cen \& Haiman 2000; Haiman 2002; Madau 2002) have recently focused on the width of the red damping wing - related to the expected strength of the Ly $\alpha$ emission line - in the spectra of very distant QSOs and star-forming galaxies with a non-negligible escape fraction of UV photons into the IGM, as a flag of the observation of the universe before re-ionization. They have assessed, in particular, the impact of the photo-ionized, hundreds of $\mathrm{kpc}$ - to Mpc-size regions which will surround individual luminous sources of Lyman-continuum radiation, on the transmission of photons redward of the Ly $\alpha$ resonance. If the source lifetime is shorter than the cosmic expansion and gas recombination timescales, the volume of ionized gas will be proportional to the total number of photons produced above $13.6 \mathrm{eV}$ that leaks into intergalactic space; the effect of this local photo-ionization is to greatly reduce the scattering opacity between the redshift of the source and the boundary of its $\mathrm{H}$ II region.

To better gauge this effect on real data, I have plotted in Figure 2 the KeckLRIS spectrum of the faint $z_{\mathrm{em}}=5.5$ quasar RD J 03011+0020 (Stern et al. 2000), 


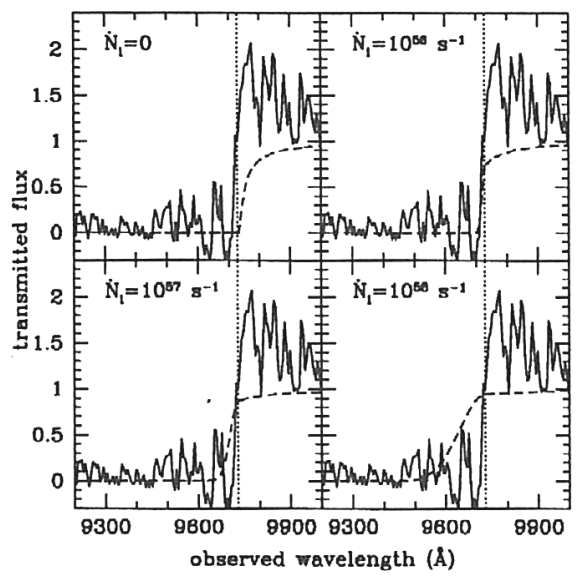

Figure 2. The Keck-LRIS spectrum of the faint $z_{\mathrm{em}}=5.5$ quasar RD J 0301117+002025 (Stern et al. 2000), redshifted to $z_{\mathrm{em}}=7$. The dotted vertical lines indicate the expected wavelength of the Ly $\alpha$ resonance. Fluxes have arbitrary normalizations. The dashed curves show the transmission through a uniform IGM, assuming the QSO is being observed prior to the re-ionization epoch at $z_{\mathrm{r}}=6$. Four different cases are shown, as the rate of emission of Lyman-continuum photons which ionize the IGM in the vicinity of the source is varied from $\dot{N}_{\mathrm{i}}=0$ to $\dot{N}_{\mathrm{i}}=10^{56}, 10^{57}$, and $10^{58} \mathrm{~s}^{-1}$. The calculations assume a quasar lifetime of $10^{7} \mathrm{yr}$. (From Madau \& Rees 2000.)

redshifted to $z_{\mathrm{em}}=7$. The Figure depicts the same $800 \AA$-wide region of the observed spectrum around the Ly $\alpha$ resonance, together with the transmission through a uniform IGM, assuming the QSO is being observed prior to the reionization epoch at $z_{\mathrm{r}}=6$. This should be taken just as an illustrative example, as in some numerical simulations (e.g., Ciardi et al. 2000) re-ionization was already well in progress prior to redshift $z=6$, and the form of the damping profile would be different in the case of patchy ionization along the line of sight. The suppression effect of the Ly $\alpha$ emission line by the wing of the GP trough, while clearly visible in the absence of a local $\mathrm{H}$ II region (top-left panel), weakens significantly as the size of the photo-ionized zone increases. On the blue side of the resonance, the transmission profile is due to the combination of two effects: (i) the damping wing - i.e., the curve in the top-left panel at $\lambda_{\mathrm{obs}}>\lambda_{\alpha}\left(1+z_{\mathrm{em}}\right)$ - shifted to the blue by the difference between the redshift of the H II region boundary and the quasar redshift; and (ii) the ordinary GP optical depth from the residual neutral gas in the $\mathrm{H}$ II zone.

Depending on the amount of $\mathrm{H}$-ionizing photons which escape the $\mathrm{H}$ I galactic layers into the intergalactic space, the red damping wing may be detectable in the spectra of star-forming early galaxies. Recently, a Ly $\alpha$ emitting galaxy has been discovered by $\mathrm{Hu}$ et al. (2002) at $z=6.56$, possibly close or prior to the re-ionization epoch. The inferred star-formation rate is $\sim 10 \mathrm{M}_{\odot} \mathrm{yr}^{-1}$. For standard initial mass functions, about 3000-4000 photons are produced above $13.6 \mathrm{eV}$ for every stellar baryon. Thus, a galaxy that turns a gas mass $M_{*}$ into stars will radiate a total of about $10^{69.6}\left(M_{*} / 10^{9} \mathrm{M}_{\odot}\right)$ Lyman-continuum 


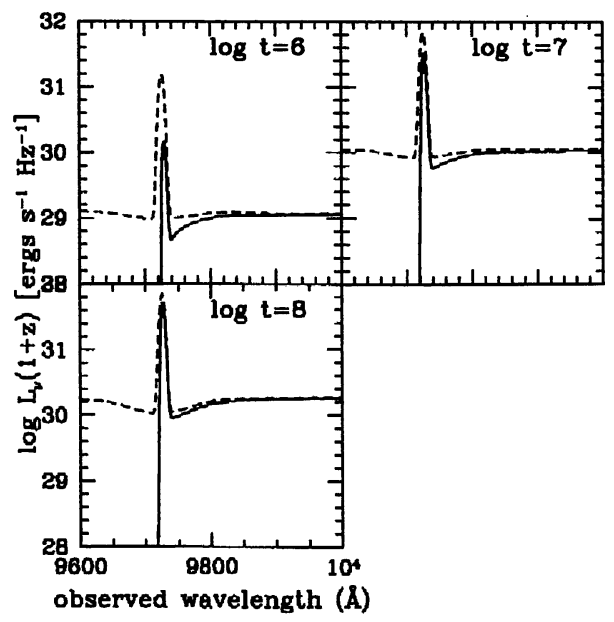

Figure 3. Dashed lines: Synthetic UV intrinsic spectra of a galaxy at $z_{\mathrm{em}}=7$ as a function of age $t$ (in yr). The stellar population has continuous star formation $\left(\dot{M}=10 \mathrm{M}_{\odot} \mathrm{yr}^{-1}\right)$ with a Salpeter IMF and metallicity $Z=0.05 \mathrm{Z}_{\odot}$. The model spectra were calculated with STARBURST99 (Leitherer et al. 1999). The Ly $\alpha$ line is computed in Case B recombination, assuming an escape fraction of Lyman-continuum photons of $50 \%$ and a Doppler-width of $150 \mathrm{~km} \mathrm{~s}^{-1}$. Solid lines: Same spectra transmitted through a uniform IGM, assuming the source is being observed prior to the re-ionization epoch (from Madau, in preparation).

photons. Figure 3 shows the expected UV spectrum and Ly $\alpha$ line profile for a luminous, star-forming galaxy observed at $z_{\mathrm{em}}=7$ prior to re-ionization, as a function of stellar age (Madau, in preparation). The escape fraction of ionizing photons into the IGM is assumed to be $50 \%$. It is clear from the figure that the overall suppression of the Ly $\alpha$ flux decreases as a function of age: in general (depending on the intrinsic line width), the emission line remains observable at late times if the escape fraction of Lyman-continuum photons exceeds 5-10\% (see also Haiman 2002). The detection of a strong Ly $\alpha$ emission line in the spectra of bright QSOs or luminous galaxies cannot then be used, by itself, as a constraint on the re-ionization epoch. The first signs of an object radiating prior to the transition from a neutral to an ionized universe may be best searched for in the spectra of transient sources like gamma-ray bursts.

\section{References}

Barkana, R., Haiman, Z., Ostriker, J.P. 2001, ApJ 558, 482

Becker, R.H., Fan, X., White, R.L., et al. 2001, AJ 122, 2850

Bullock, J.S., Kravtsov, A.V., Weinberg, D.H. 2000, ApJ 539, 517

Cen, R., Haiman, Z. 2000, ApJ (Letters) 542, L75

Cen, R., Miralda-Escudé, J., Ostriker, J.P., Rauch, M. 1994, ApJ (Letters) 437, L9

Chiu, W.A., Ostriker, J.P. 2000, ApJ 534, 507

Ciardi, B., Ferrara, A., Governato, F., Jenkins, A. 2000, MNRAS 314, 611 
Dekel, A., Silk, J. 1986, ApJ 303, 39

Djorgovski, S.G., Castro, S.M., Stern, D., Mahabal, A.A. 2001, ApJ (Letters) 560, L5

Fan, X., Strauss, M.A., Schneider, D.P., Gunn, J.E., et al. 2001, AJ 121, 54

Fukugita, M., Hogan, C.J., Peebles, P.J.E. 1998, ApJ 503, 518

Gunn, J.E., Peterson, B.A. 1965, ApJ 142, 1633

Haardt, F., Madau, P. 1996, ApJ 461, 20

Haiman, Z., Loeb, A. 1998, ApJ 503, 505

Haiman, Z. 2002, ApJ (Letters) 576, 1

Hernquist, L., Katz, N., Weinberg, D., Miralda-Escudé, J. 1996, ApJ (Letters) 457, L51

Hu, E.M., Cowie, L.L., McMahon, R.G., et al. 2002, ApJ (Letters) 568, L75

Kriss, G.A., Shull, J.M., Oegerle, W., et al. 2001, Science 293, 1112

Leitherer, C., Schaerer, D., Goldader, J.D., et al. 1999, ApJS 123, 3

Mac Low, M.-M., Ferrara, A. 1999, ApJ 513, 142

Madau, P., Haardt, F., Rees, M.J. 1999, ApJ 514, 648

Madau, P., Rees, M.J. 2000, ApJ (Letters) 542, L69

Madau, P., Ferrara, A., Rees, M.J. 2001, ApJ 555, 92

Miralda-Escudé, J. 1998, ApJ 501, 15

Moore, B., Ghigna, S., Governato, F., Lake, G., Quinn, T., Stadel, J., Tozzi, P. 1999, ApJ (Letters) 524, L19

Mori, M., Ferrara, A., Madau, P. 2002, ApJ 571, 40

Scannapieco, E., Ferrara, A., Madau, P. 2002, ApJ 574, 590

Shapiro, P.R., Giroux, M.L. 1987, ApJ (Letters) 321, L107

Shaver, P.A., Wall, J.V., Kellerman, K.I., Jackson, C.A., Hawkins, M.R.S. 1996, Nature 384, 439

Steidel, C.C., Pettini, M., Adelberger, K.L. 2001, ApJ 546, 665

Stern, D., Spinrad, H., Eisenhardt, P., Bunker, A.J., Dawson, S., Stanford, S.A., Elston, R. 2000, ApJ (Letters) 533, L75

Tegmark, M., Silk, J., Evrard, A. 1993, ApJ 417, 54

White, S.D.M., Rees, M.J. 1978, MNRAS 183, 341

Zhang, Y., Anninos, P., Norman, M.L. 1995, ApJ (Letters) 453, L57

\section{Discussion}

Pettrini: That was a great talk, Piero, and I apologize for asking such a simple question, but incidentally, what would happen to Ly $\alpha$ emission at high $z$ if the escape fraction of Ly-continuum photons was closer to $10 \%$ (the value measured at $z=3.5$ ) than the $50 \%$ you have assumed?

MADAU: Thank you, Max. Of course, as the escape fraction of ionizing photons into the IGM drops, the opacity due to the red damping wing of the Gunn-Peterson trough increases, but also more ionizing photons are turned into Ly $\alpha$. Overall, I find that star-forming galaxies with star-formation rates in excess of a few $\mathrm{M}_{\odot} \mathrm{yr}^{-1}$ and escape fractions $\geq 10 \%$ should be detectable as Ly $\alpha$ emitters prior to the re-ionization epoch. Incidentally, I thought the escape fraction you guys measured from LBGs at $z=3.5$ was closer to $50 \%$.

KUDRITZKI: Massive PopIII stars should produce enormous Ly $\alpha$ recombination lines, and it would be great if we were able to observe those. How stable are your calculations predicting that Ly $\alpha$ could overcome the red wing Gunn-Peterson absorption? It must 
depend on the fraction of leaking Ly-continuum photons.

MADAU: I used metallicities $Z=0.05 \mathrm{Z}_{\odot}$ in my calculations of the effect of the red damping wing of the Gunn-Peterson trough on Ly $\alpha$ emitters prior to the re-ionization epoch. I plan to redo these calculations also for $Z=0$ Pop III spectra. But you are right, the most important parameter is the escape fraction of Ly-continuum photons into the IGM.

ZINNECKER: In the beginning of your talk, did I hear you saying that the second generation of Pop III stars formed from gas with $\sim 10^{4} \mathrm{~K}$ ?

MADAU: I used the term 'second generation' only as an illustration. There is a 'minimum' mass threshold for baryonic condensation in cosmology, $M_{\min } \approx 5 \times 10^{5} \mathrm{M}_{\odot}$. Above $M_{\min }$ the $\mathrm{H}_{2}$ cooling time is shorter than the Hubble time at virialization, the gas in the central halo regions becomes self-gravitating, and stars can form. The 'first' generation of stars in the universe formed in halos just above $M_{\min }$ (possibly with a top-heavy IMF). I termed 'second' generation those stars that formed at later times in halos with virial temperatures $T_{\text {vir }} \geq 10^{4} \mathrm{~K}$, or equivalently with masses $M \geq 10^{9}(1+Z)^{-3 / 2} h^{-1} \mathrm{M}_{\odot}$. In these more massive halos the gas can cool catastrophically, because of the excitation of hydrogen Ly $\alpha$ by the Maxwellian tail of the electron distribution, and perhaps fragmented to a more 'normal' IMF.

KAPER: What is the current status of our knowledge of the cosmic star-formation rate as a function of redshift (the 'Madau' plot)? Can you predict the GRB rate as a function of redshift?

MADAU: We have very little knowledge of the universe at $z>4$, and in particular of the galaxy population, star-formation rate per unit volume, and GRB rate at these early epochs. Observation will improve fast in the near future: the HST-ACS Treasury Survey in the GOODS fields will soon provide new constraints on color-selected $B, V$ and $I$-'dropouts' at $3.5<z<6.5$. And the Swift satellite should observe hundreds of GRB afterglows and measure their redshift distribution and cosmological evolution. At lower redshifts, Porciani \& Madau (2001, ApJ 548, 522) have made predictions of GRB rates based on a set of plausible star formation histories. But uncertainties in the luminosity function of GRBs make it difficult to use the observed burst number counts to constrain the rate of formation of massive stars in the universe.

SONNEBORN: What is the fate of the small dark matter halos in the Local Group?

MADAU: A large number of them is expected to survive tidal disruption. If re-ionization does make them really 'dark' (in the sense of inhibiting star-formation), then the only way of detecting these substructures 'directly' is through gravitational lensing. Recently, a number of people (Metcalf, Kochanek, Zhao, Dalal, Keeton, and myself) have argued that the anomalous image flux ratios observed in several lenses could be explained by substructures such as low mass dark matter satellites in the primary lens galaxy. 